\title{
Ramsey number of a connected triangle matching
}

\author{
András Gyárfás* \\ Alfréd Rényi Institute of Mathematics \\ Hungarian Academy of Sciences \\ Budapest, P.O. Box 127 \\ Budapest, Hungary, H-1364 \\ gyarfas.andras@renyi.mta.hu
}

\author{
Gábor N. Sárközy ${ }^{\dagger}$ \\ Alfréd Rényi Institute of Mathematics \\ Hungarian Academy of Sciences \\ Budapest, P.O. Box 127 \\ Budapest, Hungary, H-1364 \\ and \\ Computer Science Department \\ Worcester Polytechnic Institute \\ Worcester, MA, USA 01609 \\ gsarkozy@cs . wpi . edu
}

September 21, 2015

\begin{abstract}
We determine the 2-color Ramsey number of a connected triangle matching $c\left(n K_{3}\right)$ which is any connected graph containing $n$ vertex disjoint triangles. We obtain that $R\left(c\left(n K_{3}\right), c\left(n K_{3}\right)\right)=7 n-2$, somewhat larger than in the classical result of Burr, Erdős and Spencer for a triangle matching, $R\left(n K_{3}, n K_{3}\right)=5 n$. The motivation is to determine the Ramsey number $R\left(C_{n}^{2}, C_{n}^{2}\right)$ of the square of a cycle $C_{n}^{2}$. We apply our Ramsey result for connected triangle matchings to show that the Ramsey number of an "almost" square of a cycle $C_{n}^{2, c}$ (a cycle of length $n$ in which all but at most a constant number $c$ of short diagonals are present) is asymptotic to $7 n / 3$.
\end{abstract}

\section{Introduction}

Denote by $\delta(G)$ the minimum degree in a graph $G . K_{n}$ is the complete graph on $n$ vertices and $K_{n, n}$ is the complete bipartite graph between two sets of $n$ vertices each. If $G_{1}, G_{2}, \ldots, G_{r}$ are graphs, then the Ramsey number $R\left(G_{1}, G_{2}, \ldots, G_{r}\right)$ is the

\footnotetext{
${ }^{*}$ Research supported in part by the OTKA Grant No. K104343.

${ }^{\dagger}$ Research supported in part by the National Science Foundation under Grant No. DMS-0968699 and by OTKA Grant No. K104343.
} 
smallest positive integer $n$ such that in any edge-coloring with colors $1,2, \ldots, r$, for some $i$ the edges of color $i$ contain a subgraph isomorphic to $G_{i}$. In this paper we will deal with 2- and 3-color Ramsey numbers (so $r=2$ or 3 ) and we will think of color 1 as red, color 2 as blue and color 3 (if it exists) as white.

Among well-known early results in generalized Ramsey theory is the exact value of $R\left(n K_{2}, n K_{2}\right)=3 n-1$ determined by Cockayne and Lorimer [8], and $R\left(n K_{3}, n K_{3}\right)=$ $5 n$ determined by Burr, Erdős and Spencer [3]. Here $n G$ denotes $n$ vertex disjoint copies of the graph $G$. It turned out in many applications that it is important to study the case when $n K_{2}$, the matching, is replaced by a connected matching, $c\left(n K_{2}\right)$, defined as any connected graph containing $n K_{2}$ (see for example applications [2, 9, 11, 12, 13, 14, 15, 16, 22]). The 2-color Ramsey number of connected matchings is the same as the Ramsey number of matchings (in fact, [10] proves more) and one of the key arguments of [13] was that this remains true for 3 colors as well. However, for more than 3 colors the Ramsey numbers of matchings and connected matchings are different. For example, $R\left(n K_{2}, n K_{2}, n K_{2}, n K_{2}\right)=5 n-3$ [8], but $R\left(c\left(n K_{2}\right), c\left(n K_{2}\right), c\left(n K_{2}\right), c\left(n K_{2}\right)\right)>6 n-3$ when $2 n-1$ is divisible by three. This can be seen by the 4-coloring obtained from the parallel classes of an affine plane of order 3 by replacing each point with a point set of size $\frac{2 n-1}{3}$.

In this paper we look at the connected version of the "matching of triangles". Let $c\left(n K_{3}\right)$ denote any connected graph containing $n$ vertex disjoint triangles. We shall prove that here already the 2-color Ramsey number of $c\left(n K_{3}\right)$ is different from its counterpart $n K_{3}$.

Theorem 1. For $n \geq 2, R\left(c\left(n K_{3}\right), c\left(n K_{3}\right)\right)=7 n-2$.

(While we have $R\left(n K_{3}, n K_{3}\right)=5 n$.) To see that $R\left(c\left(n K_{3}\right), c\left(n K_{3}\right)\right)>7 n-3$, consider pairwise disjoint sets $A, B, C$ such that $V\left(K_{7 n-3}\right)=A \cup B \cup C,|A|=|B|=3 n-1$ and $|C|=n-1$. Edges inside $A$ and inside $B$ are red, all other edges are blue. In this coloring there is no monochromatic $c\left(n K_{3}\right.$ ) (in fact there is not even an $n K_{3}$ in blue).

To prove that $R\left(c\left(n K_{3}\right), c\left(n K_{3}\right)\right) \leq 7 n-2$, we need the Ramsey number of connected triangle matchings versus ordinary matchings that might be interesting on its own.

Lemma 2. For $1 \leq m \leq n, R\left(c\left(n K_{3}\right), m K_{2}\right)=3 n+m-1$.

Notice that $R\left(c\left(n K_{3}\right), m K_{2}\right)>3 n+m-2$ is shown by the disjoint sets $A, X$ such that $V\left(K_{3 n+m-2}\right)=A \cup X,|A|=3 n-1,|X|=m-1$ and edges inside $A$ are colored red, other edges are colored with blue. In this coloring there is no red $n K_{3}$ or blue $m K_{2}$. 
The motivation of Theorem 1 comes from the effort to determine or estimate the 2-color Ramsey number $R\left(C_{n}^{2}, C_{n}^{2}\right)$ where of $C_{n}^{2}$ is the square of the cycle on $n$ vertices, i.e. the cycle $C_{n}$ with all short diagonals (diagonals between vertices at a distance 2 on the cycle). A very recent paper of Allen, Brightwell and Skokan [1] gives lower bound $3 n-4$ and upper bound $\frac{20 n}{3}+o(n)$ for that Ramsey number.

Density questions for the square of a cycle have also received a lot of attention (for example the well-known Pósa-Seymour problem, see [4], [17, 21]). Note also that there has been a lot of research on the Ramsey numbers of constant maximum degree graphs (such as $C_{n}^{2}$, where the maximum degree is 4). It is known that for a graph $G$ on $n$ vertices with maximum degree $\Delta$ the Ramsey number is linear, it is at most $c(\Delta) n$ [5]. The current best bound $c(\Delta) \leq 2^{c \Delta \log \Delta}$ is due to Conlon, Fox and Sudakov [6].

To determine $R\left(C_{n}^{2}, C_{n}^{2}\right)$ exactly for every $n$ is hopeless since for $n=5,6, C_{5}^{2}=$ $K_{5}, C_{6}^{2}=K_{6}-3 K_{2}$ and these Ramsey numbers are both unknown. Also, the constant $c(4)$ in the linear bounds $c(4) n$ is very large. However, combining Theorem 1 with the Regularity Lemma, we shall make a step forward and prove the following. Let $C_{n}^{2, c}$ denote an "almost" square of the cycle $C_{n}$, a cycle of length $n$ in which all but at most a constant number $c$ of short diagonals are present.

Theorem 3. For every fixed $\eta>0$ there is a $c=c(\eta)$ so that for any $n \geq c$ we have $R\left(C_{n}^{2, c}, C_{n}^{2, c}\right) \leq(1+\eta) 7 n / 3$.

It is worth noting that Theorem 3, although asymptotically sharp (shown by a similar construction as in Theorem 11), does not give the right asymptotics for $R\left(C_{n}^{2}, C_{n}^{2}\right)$, where we insist on all short diagonals. Indeed, $R\left(C_{n}^{2}, C_{n}^{2}\right) \geq 3 n-4$ is proved in [1]. Thus perhaps surprisingly removing these constant number of diagonals makes a big difference in the Ramsey number.

The following easy lemma from [15] will be used. It extends (when $\delta(G)=$ $|V(G)|-1)$ the well-known remark of Erdös and Rado that in a 2-colored complete graph there is a monochromatic spanning tree.

Lemma 4. (Lemma 1.5 in [15]) Suppose that the edges of a graph $G$ with $\delta(G) \geq$ $\frac{3|V(G)|}{4}$ are 2 -colored. Then there is a monochromatic connected subgraph with order larger than $\delta(G)$. This estimate is sharp.

Theorem 1 and Lemma 2 are proved in Section 2. Their perturbed versions are worked out in Section 3. Section 4 outlines the (rather standard) argument how to obtain Theorem 3 from the Regularity and Blow-up lemmas. 


\section{The proof of Lemma 2 and Theorem 1}

Proof of Lemma 2. We prove by induction on $m$. Since for $m=1$ the statement is trivially true for every $n$, suppose we have a blue matching $M=(m-1) K_{2}$ in a 2-coloring of a $K_{3 n+m-1}$ with vertex set $V$. If there is no blue $m K_{2}$ then every edge $e_{i} \in M$ has a vertex $p_{i}$ adjacent in red to all but at most one vertex of $X=V-V(M)$ (otherwise $e_{i}$ could be replaced by two independent blue edges). Also, $X$ induces a red complete graph. Since

$$
|X|=3 n+m-1-2(m-1)=3 n-m+1 \geq 2 m+1,
$$

we can select greedily $m-1$ pairwise disjoint red triangles with one vertex as $p_{i}$ and two vertices from $X$. Then we find red triangles greedily in the remainder of $X$. We are guaranteed to find $n$ red triangles this way. These triangles can be certainly included into a connected red subgraph so we have the required $c\left(n K_{3}\right)$.

Proof of Theorem 1. Consider a 2-coloring of the edges of $K=K_{7 n-2}$ with red and blue, assume w.l.o.g. that the blue color class has only one connected component (since one color class is connected by using Lemma 4 with $\delta(G)=|V(G)|-1$ ). Since $R\left(n K_{3}, n K_{3}\right)=5 n$ for $n \geq 2$, we have a monochromatic $n K_{3}$. If it is blue, we are done, therefore it is red and the red color class must define at least two connected components. Suppose that the red components have vertex sets $X_{1}, X_{2}, \ldots, X_{s}$, where $s \geq 2$ and $\left|X_{1}\right| \geq\left|X_{2}\right| \geq \ldots \geq\left|X_{s}\right| \geq 1$. We may suppose that $\left|X_{1}\right| \leq 5 n$, otherwise we have the required monochromatic $c\left(n K_{3}\right)$ from $R\left(n K_{3}, n K_{3}\right)=5 n$.

Case 1. $\left|X_{1}\right| \geq\left|X_{2}\right| \geq 3 n,\left|X_{1}\right|=3 n+k_{1},\left|X_{2}\right|=3 n+k_{2}$. Since by Lemma 2 we have $R\left(c\left(n K_{3}\right),\left(k_{i}+1\right) K_{2}\right)=3 n+k_{i}$ for $i=1,2, X_{i}$ contains either a red $c\left(n K_{3}\right)$ or a blue $\left(k_{i}+1\right) K_{2}$ and we are done if the first possibility appears. Thus we have blue matchings $M_{i}$ of size $k_{i}+1$ in $X_{i}$ for $i=1,2$. We can take $k_{1}+1$ vertices in $X_{2} \backslash M_{2}$ and $k_{2}+1$ vertices in $X_{1} \backslash M_{1}$ to form a blue $T=\left(k_{1}+k_{2}+2\right) K_{3}$ using the blue edges between $X_{1}$ and $X_{2}$ since from $k_{1}+k_{2} \leq n-2$ it follows that

$$
\begin{aligned}
& 3 n+k_{1}-2\left(k_{1}+1\right)=3 n-k_{1}-2 \geq k_{2}+1, \\
& 3 n+k_{2}-2\left(k_{2}+1\right)=3 n-k_{2}-2 \geq k_{1}+1 .
\end{aligned}
$$

If $k_{1}+k_{2}+2=n$ (i.e. $s=2$ ) we have the required blue $c\left(n K_{3}\right)$. Otherwise we have $l=n-\left(k_{1}+k_{2}\right)-2(>0)$ vertices in $A=V-\left(X_{1} \cup X_{2}\right)$ and we can form $l$ vertex disjoint blue triangles taking one vertex from each of the sets $A, X_{1}-T, X_{2}-T$. We have enough vertices for that, because

$$
\left|X_{1}-T\right|=3 n+k_{1}-2\left(k_{1}+1\right)-\left(k_{2}+1\right)=3 n-\left(k_{1}+k_{2}\right)-3 \geq n-\left(k_{1}+k_{2}\right)-2
$$


and the same is true for $\left|X_{2}-T\right|$. Thus we have a connected blue triangle matching of size at least $k_{1}+k_{2}+2+n-\left(k_{1}+k_{2}\right)-2=n$, as desired finishing Case 1 .

Case 2. $\left|X_{1}\right| \geq 3 n, 2 n \leq\left|X_{2}\right|<3 n,\left|X_{1}\right|=3 n+k_{1},\left|X_{2}\right|=3 n-k_{2}, 1 \leq k_{2} \leq n$. Again since by Lemma 2 we have $R\left(c\left(n K_{3}\right),\left(k_{1}+1\right) K_{2}\right)=3 n+k_{1}$, we may suppose that we have a blue $M_{1}=\left(k_{1}+1\right) K_{2}$ in $X_{1}$. We transform $M_{1}$ to a blue triangle matching $T=\left(k_{1}+1\right) K_{3}$ using $k_{1}+1$ vertices from $X_{2}$ and then extend $T$ using $q=n-k_{1}+k_{2}-2$ vertices in $A=V-\left(X_{1} \cup X_{2}\right)$ and $q$ vertices from both $X_{1}, X_{2}$. We have enough room for that because

$$
3 n+k_{1}-2\left(k_{1}+1\right) \geq n-k_{1}+k_{2}-2,3 n-k_{2}-\left(k_{1}+1\right) \geq n-k_{1}+k_{2}-2
$$

are both true since $k_{2} \leq n$. Thus we have a connected blue triangle matching of size at least $\left(k_{1}+1\right)+\left(n-k_{1}+k_{2}-2\right)=n+k_{2}-1 \geq n$.

Case 3. $\left|X_{1}\right| \geq 3 n, n \leq\left|X_{2}\right|<2 n,\left|X_{1}\right|=3 n+k_{1},\left|X_{2}\right|=n+k_{2}, 0 \leq k_{2}<n$. Again since by Lemma 2 we have $R\left(c\left(n K_{3}\right),\left(k_{1}+1\right) K_{2}\right)=3 n+k_{1}$, we may suppose that we have a blue $M_{1}=\left(k_{1}+1\right) K_{2}$ in $X_{1}$. Furthermore, we may suppose that $k_{1}<n$ otherwise $M_{1}$ can be transformed to a blue triangle matching $T=\left(k_{1}+1\right) K_{3}$ using vertices from $X_{2}$. Since $k_{2}<n$ also holds, $\left|V-\left(X_{1} \cup X_{2}\right)\right|=3 n-\left(k_{1}+k_{2}\right)-2 \geq n$. Thus we have at least $n$ vertices in all of the three sets $X_{1}, X_{2}, V-\left(X_{1} \cup X_{2}\right)$ implying that we have a connected blue $c\left(n K_{3}\right)$.

Case 4. $\left|X_{1}\right| \geq 3 n,\left|X_{2}\right|<n$ or $\left|X_{1}\right|<3 n$.

From Lemma 2 we may assume $\left|X_{1}\right| \leq 4 n-2$. Indeed, otherwise since by Lemma 2 we have $R\left(c\left(n K_{3}\right), n K_{2}\right)=4 n-1$, we may suppose that we have a blue $M_{1}=n K_{2}$ in $X_{1}$. This blue $M_{1}$ can be transformed into a blue $c\left(n K_{3}\right)$ using $n$ vertices in $V-X_{1}$ $\left(\left|X_{1}\right|<5 n\right.$ ensures that there are $n$ vertices $)$.

Define the set $S_{1}$ so that $\left|S_{1}\right|=n$ and, starting with $X_{1}$, all vertices of $X_{i}$ are selected before taking vertices from $X_{i+1}$. Then, starting from the next $X_{i}$, define $S_{2}$ in the same way. Now set

$$
A=\left\{\cup X_{i}:\left(S_{1} \cup S_{2}\right) \cap X_{i}=\emptyset\right\} .
$$

Then we have the following claim.

$$
|A| \geq n \text { (or equivalently }|V \backslash A| \leq 6 n-2 \text { ) }
$$

Indeed, this is true either because $\left|X_{1}\right| \leq 4 n-2$ and $\left|X_{2}\right|<n$ so the last $X_{i}$ which intersects $S_{2}$ satisfies $\left|X_{i}\right|<n$ or because $3 n>\left|X_{1}\right| \geq\left|X_{2}\right|$. But then we can select $S_{3} \subset A$ with $\left|S_{3}\right|=n$, and the blue complete tripartite graph $\left[S_{1}, S_{2}, S_{3}\right]$ defines the required blue $c\left(n K_{3}\right)$. 


\section{Perturbed version of Theorem 1}

As in many applications of the Regularity Lemma, one has to handle a few irregular pairs and the corresponding edges will not be present in the reduced graph. We say that the graph $G$ on $n$ vertices is $\varepsilon$-perturbed if it is almost complete, at most $\varepsilon\left(\begin{array}{l}n \\ 2\end{array}\right)$ edges are missing. We cannot apply Theorem 1 in the reduced graph because in Theorem 1 we have a 2-colored complete graph, yet the reduced graph will be a 2 -colored $\varepsilon$-perturbed graph. Thus we need perturbed versions of Theorem 1 and first Lemma 2. It will be convenient to think of the missing edges as edges in a third color class (white or "invisible"), so we move up from 2-color Ramsey numbers to 3-color Ramsey numbers. $K_{1, t}$ denotes the star with $t$ leaves.

Lemma 5. For $1 \leq m \leq n, 0 \leq t \leq n, R\left(c\left(n K_{3}\right), m K_{2}, K_{1, t}\right) \leq 3 n+m-1+2 t$.

Proof. We prove by induction on $m$ as in the non-perturbed case. The starting case, $m=1$ follows easily from a well-known result of Corradi and Hajnal [7] (or it could also be proved directly by an easy induction on $n$ ). Indeed, if there is no blue edge, we have a red graph on $N=3 n+2 t$ vertices with minimum degree at least $3 n+t>\frac{2 N}{3}$ and it contains at least $\lfloor N / 3\rfloor \geq n$ vertex disjoint red triangles. Since our red graph is automatically connected from the minimum degree condition, we have the required red $c\left(n K_{3}\right)$. Thus, we may select a blue matching $M=(m-1) K_{2}$ in a 2-coloring of a $K_{3 n+m-1+2 t}$ with vertex set $V$. We may assume that from every vertex fewer than $t$ edges are missing (or white edges). If there is no blue $m K_{2}$ then every edge $e_{i} \in M$ again has a vertex $p_{i}$ that is adjacent in blue to at most one vertex in $X=V-V(M)$. However, now $p_{i}$ is not necessarily adjacent in red to all other vertices in $X$ since some edges might be missing. But all the edges that are actually present are indeed red to the other vertices. Furthermore, in $X$ all edges that are present are red as well. Since

$$
|X|=3 n+m-1+2 t-2(m-1)=3 n-m+1+2 t \geq 2 m+1+2 t,
$$

again we can select greedily $m-1$ pairwise disjoint red triangles with one vertex as $p_{i}$ and two vertices from $X$. Indeed, $p_{i}$ is still adjacent in red to more than $(2 t+3)-t=t+3>t$ vertices in $X$ but then there is a (red) edge among these neighbors, giving a red triangle as desired. Then we find red triangles greedily in the remainder of $X$ similarly. Finally, we find the $n$-th red triangle in the remainder of $X$ as follows. Select an arbitrary remaining vertex of $X$. Since it has more than $t$ neighbors left in $X$, there is an edge among these neighbors and all edges are red in $X$. The red graph spanned by $X$ is connected because $|X| / 2>t$, thus the $n$ red triangles form a $c\left(n K_{3}\right)$.

We will also need a perturbed version of the classical result of Burr, Erdös and Spencer, $R\left(n K_{3}, n K_{3}\right)=5 n$. 
Lemma 6. For $n \geq 2,1 \leq t \leq n, R\left(n K_{3}, n K_{3}, K_{1, t}\right) \leq 6 n-2+5 t$.

Proof. Consider the largest blue triangle matching, remove it, then consider the largest red triangle matching in the remainder and remove it. We have at least $5 t+4$ vertices left and there are no more monochromatic triangles. However, consider an arbitrary vertex, it is still adjacent to at least $4 t+4$ vertices in the leftover. Then in one of the colors (say blue) it is adjacent to at least $2 t+2$ vertices. These neighbors will induce a triangle which must be red (otherwise we get a blue triangle), a contradiction. Indeed, consider again an arbitrary vertex from these at least $2 t+2$ vertices, it is still adjacent to at least $t+2$ vertices from these at least $2 t+2$ vertices. But then there must be an edge within these at least $t+2$ vertices, giving a triangle.

We note that more is true $(5 n+c t)$ but for our purposes this weaker statement is sufficient.

Next we are ready to give the perturbed version of Theorem 1 .

Theorem 7. For $n \geq 2,0 \leq t \leq 2 n / 3, R\left(c\left(n K_{3}\right), c\left(n K_{3}\right), K_{1, t}\right) \leq 7 n-2+7 t$.

Proof. Again suppose we have a 2-coloring of a $K_{7 n-2+7 t}$ with vertex set $V$. We may assume that from every vertex fewer than $t$ edges are missing (edges in the third color). Applying Lemma 4, we get a monochromatic (say blue) connected component $X$ of size at least $(7 n-2+7 t)-t=7 n-2+6 t$. By Lemma 6, since $7 n-2+6 t \geq 6 n-2+5 t$, we have a monochromatic $n K_{3}$ in $X$. If it is blue, we are done, therefore it is red and thus the red color class must define at least two connected components within $X$. Suppose that the red components of $V$ have vertex sets $X_{1}, X_{2}, \ldots, X_{s}$, where $s \geq 2$ and $\left|X_{1}\right| \geq\left|X_{2}\right| \geq \ldots \geq\left|X_{s}\right| \geq 1$. We may suppose that $\left|X_{1}\right| \leq 6 n-2+5 t$ otherwise we have the required monochromatic $c\left(n K_{3}\right)$ from Lemma 6 .

Case 1. $\left|X_{1}\right| \geq\left|X_{2}\right| \geq 3 n+2 t,\left|X_{1}\right|=3 n+2 t+k_{1},\left|X_{2}\right|=3 n+2 t+k_{2}$. Here we apply Lemma 5 to the two subgraphs induced by $X_{1}$ and $X_{2}$. We find either a red $c\left(n K_{3}\right)$ or a blue $\left(k_{i}+1\right) K_{2}$ in them and we are done if the first possibility appears. Thus we have blue matchings $M_{1}, M_{2}$ of size $k_{1}+1, k_{2}+1$, respectively.

We extend $M_{1}$ to a blue $\left(k_{1}+1\right) K_{3}$ by taking $k_{1}+1$ vertices in $X_{2}-M_{2}$. This can be done if $\left|X_{2}-M_{2}\right| \geq k_{1}+1+2 t-2$, extending the edges of $M_{1}$ one by one to blue triangles, at each step we have at most $2 t-2$ vertices in $X_{2}-M_{2}$ not adjacent (in blue) to at least one of the ends of the edge to be extended. Indeed,

$$
\left|X_{2}-M_{2}\right|=3 n+2 t+k_{2}-2\left(k_{2}+1\right)=3 n+2 t-k_{2}-2 \geq k_{1}+1+2 t-2
$$

i.e $3 n \geq k_{1}+k_{2}+1$ which is true since $k_{1}+k_{2}+1 \leq n+3 t-1 \leq n+2 n-1$ from the assumption $t \leq 2 n / 3$. The same argument allows to extend $M_{2}$ to a blue $\left(k_{2}+1\right) K_{3}$ with $k_{2}+1$ vertices of $X-M_{1}$. Thus we have a blue $T=\left(k_{1}+k_{2}+2\right) K_{3}$ and noticing 
that the blue graph spanned by $X_{1} \cup X_{2}$ is connected (by $\left|X_{1}\right|,\left|X_{2}\right|>2 t$, any two vertices of $X_{1}$ and of $X_{2}$ has a common blue neighbor) we are done if $k_{1}+k_{2}+2 \geq n$.

Otherwise we have $l=n+3 t-\left(k_{1}+k_{2}\right)-2(>0)$ vertices in $A=V-\left(X_{1} \cup X_{2}\right)$ and we plan to extend $T$ to $n K_{3}$ with $n-\left(k_{1}+k_{2}+2\right)$ vertex disjoint blue triangles taking one vertex from each of the sets $A, X_{1}-T, X_{2}-T$. Since $T$ is already connected, the extension will be automatically connected as well. We have enough vertices for that if all the three sets have size at least $n-\left(k_{1}+k_{2}+2\right)+2 t$ (in fact two of them can be only at least $\left.n-\left(k_{1}+k_{2}+2\right)+t\right)$. In our case the condition holds for $A$ with $t$ to save and for $X_{i}-T$ it holds with about $2 n$ to save:

$$
\begin{aligned}
& \left|X_{1}-T\right|=3 n+2 t+k_{1}-2\left(k_{1}+1\right)-\left(k_{2}+1\right)= \\
& =3 n+2 t-\left(k_{1}+k_{2}\right)-3 \geq n-\left(k_{1}+k_{2}+2\right)+2 t
\end{aligned}
$$

and the same is true for $\left|X_{2}-T\right|$. Thus we have a blue $c\left(n K_{3}\right)$.

Case 2. $\left|X_{1}\right| \geq 3 n+2 t, 2 n+2 t \leq\left|X_{2}\right|<3 n+2 t,\left|X_{1}\right|=3 n+2 t+k_{1},\left|X_{2}\right|=3 n+2 t-k_{2}$, $1 \leq k_{2} \leq n$.

Here we apply Lemma 5 to the subgraph induced by $X_{1}$. We may suppose we have a blue $M_{1}=\left(k_{1}+1\right) K_{2}$ in $X_{1}$, we transform $M_{1}$ to a blue triangle matching $T=$ $\left(k_{1}+1\right) K_{3}$ using $k_{1}+1$ vertices from $X_{2}$ and then extend $T$ using $q=n-k_{1}+k_{2}-2$ vertices in $A=V-\left(X_{1} \cup X_{2}\right)$ and $q$ vertices from both $X_{1}, X_{2}$. We have enough room for that because $|A|=n+3 t-k_{1}+k_{2}-2 \geq q+2 t$ and

$$
3 n+2 t+k_{1}-2\left(k_{1}+1\right) \geq q+2 t, 3 n+2 t-k_{2}-\left(k_{1}+1\right) \geq q+2 t
$$

are both true since $k_{2} \leq n$. Thus we have a connected blue triangle matching of size at least $\left(k_{1}+1\right)+q=n+k_{2}-1 \geq n$.

Case 3. $\left|X_{1}\right| \geq 3 n+2 t, n+2 t \leq\left|X_{2}\right|<2 n+2 t,\left|X_{1}\right|=3 n+2 t+k_{1},\left|X_{2}\right|=n+2 t+k_{2}$, $0 \leq k_{2}<n$.

Again we apply Lemma 5 to the subgraph induced by $X_{1}$ and select the blue $M_{1}=$ $\left(k_{1}+1\right) K_{2}$ in $X_{1}$. We may suppose that $k_{1}<n$ otherwise $M_{1}$ can be transformed to a blue triangle matching $T=\left(k_{1}+1\right) K_{3}$ using vertices from $X_{2}$. Since $k_{2}<n$ also holds, $\left|V-\left(X_{1} \cup X_{2}\right)\right|=3 n+3 t-\left(k_{1}+k_{2}\right)-2 \geq n+2 t$. Thus we have at least $n+2 t$ vertices in all of the three sets $X_{1}, X_{2}, V-\left(X_{1} \cup X_{2}\right)$ implying that we have a connected blue $c\left(n K_{3}\right)$.

Case 4. $\left|X_{1}\right| \geq 3 n+2 t,\left|X_{2}\right|<n+2 t$ or $\left|X_{1}\right|<3 n+2 t$.

We may assume $\left|X_{1}\right| \leq 4 n-2+2 t$, otherwise we can apply Lemma 5 with $m=n$ to $X_{1}$ to find a blue $n K_{2}$ and, since from Lemma $7\left|X_{1}\right| \leq 6 n-2+5 t$ we have at least $7 n-2+7 t-(6 n-2+5 t)=n+2 t$ vertices in $V-X_{1}$, the blue $n K_{2}$ can be transformed into a blue $c\left(n K_{3}\right)$ using $n$ vertices of $V-X_{1}$. 
If $\left|X_{1}\right| \geq n+2 t$, take an $(n+2 t)$-vertex subset $S_{1} \subset X_{1}$ then take an $(n+2 t)$-vertex set $S_{2}$ from $\cup_{i>1} X_{i}$ so that in $S_{2}$ we use all vertices of $X_{i}$ before taking vertices from $X_{i+1}$. Define

$$
A=\left\{\cup_{i>1} X_{i}: S_{2} \cap X_{i} \neq \emptyset\right\}
$$

Then $\left|X_{1} \cup A\right| \leq 6 n-2+6 t$ either because $\left|X_{1}\right| \leq 4 n-2+2 t$ and $\left|X_{2}\right|<n+2 t$ so the last $X_{i}$ which intersects $S_{2}$ satisfies $\left|X_{i}\right|<n+2 t$ or because $3 n+2 t>\left|X_{1}\right| \geq\left|X_{2}\right|$. Thus we can select $S_{3} \subset V-A$ with $\left|S_{3}\right| \geq n+t$, and the blue tripartite graph $\left[S_{1}, S_{2}, S_{3}\right]$ has lower bounds $n+2 t, n+2 t, n+t$ for its vertex classes which allows to pick the vertices of the required blue $c\left(n K_{3}\right)$.

If $\left|X_{1}\right|<n+2 t$, define $S_{1}$ so that $\left|S_{1}\right|=n+t$ and all vertices of $X_{i}$ are selected before taking vertices from $X_{i+1}$. Then, starting from the next $X_{i}$, define $S_{2}$ in the same way. Now set

$$
B=\left\{\cup X_{i}:\left(S_{1} \cup S_{2}\right) \cap X_{i} \neq \emptyset\right\}
$$

and observe that $|B| \leq 4 n+6 t$ thus we can select $S_{3} \subset V-B$ with $\left|S_{3}\right| \geq 7 n-2+$ $7 t-(4 n+6 t)=3 n-2+t \geq n+2 t$, and the blue complete tripartite graph $\left[S_{1}, S_{2}, S_{3}\right]$ has lower bounds $n+t, n+t, n+2 t$ for its vertex classes which allows to pick the vertices of the required blue $c\left(n K_{3}\right)$.

\section{Proof of Theorem 3; applying the Regularity Lemma}

Next we show how to prove Theorem 3 from Theorem 7 , the Regularity Lemma [23] and the Blow-up Lemma. The material of this section is fairly standard by now (see [2, 11, 12, 13, 14, 15, 16] for similar techniques) so we omit some of the details. In particular in [13] Section 2 follows a similar outline.

Let $e(X, Y)$ denote the number of edges between $X$ and $Y$ in a graph $G$. For disjoint $X, Y$, we define the density

$$
d(X, Y)=\frac{e(X, Y)}{|X| \cdot|Y|}
$$

For two disjoint subsets $A, B$ of $V(G)$, the bipartite graph with vertex set $A \cup B$ which has all the edges of $G$ with one endpoint in $A$ and the other in $B$ is called the pair $(A, B)$.

A pair $(A, B)$ is $\varepsilon$-regular if for every $X \subset A$ and $Y \subset B$ satisfying

$$
|X|>\varepsilon|A| \text { and }|Y|>\varepsilon|B|
$$

we have

$$
|d(X, Y)-d(A, B)|<\varepsilon
$$


A pair $(A, B)$ is $(\varepsilon, \delta)$-super-regular if it is $\varepsilon$-regular and furthermore,

$$
\begin{aligned}
& \quad \operatorname{deg}(a) \geq \delta|B| \quad \text { for all } a \in A, \\
& \text { and } \operatorname{deg}(b) \geq \delta|A| \text { for all } b \in B \text {. }
\end{aligned}
$$

We need a 2-edge-colored version of the Szemerédi Regularity Lemma 1

Lemma 8. For every integer $m_{0}$ and positive $\varepsilon$, there is an $M_{0}=M_{0}\left(\varepsilon, m_{0}\right)$ such that for $n \geq M_{0}$ the following holds. For any n-vertex graph $G$, where $G=G_{1} \cup G_{2}$ with $V\left(G_{1}\right)=V\left(G_{2}\right)=V$, there is a partition of $V$ into $\ell+1$ clusters $V_{0}, V_{1}, \ldots, V_{\ell}$ such that

- $m_{0} \leq \ell \leq M_{0},\left|V_{1}\right|=\left|V_{2}\right|=\ldots=\left|V_{\ell}\right|,\left|V_{0}\right|<\varepsilon n$,

- apart from at most $\varepsilon\left(\begin{array}{l}\ell \\ 2\end{array}\right)$ exceptional pairs, all pairs $\left.G_{s}\right|_{V_{i} \times V_{j}}$ are $\varepsilon$-regular, where $1 \leq i<j \leq \ell$ and $1 \leq s \leq 2$.

Our other main tool is the Blow-up Lemma (see [18, 19]). It basically says that super-regular pairs behave like complete bipartite graphs from the point of view of bounded degree subgraphs.

Lemma 9. Given a graph $R$ of order $r$ and positive parameters $\delta, \Delta$, there exists an $\varepsilon>0$ such that the following holds. Let $m$ be an arbitrary positive integer, and let us replace the vertices of $R$ with pairwise disjoint $m$-sets $V_{1}, V_{2}, \ldots, V_{r}$ (blowing up). We construct two graphs on the same vertex-set $V=\cup V_{i}$. The graph $R(m)$ is obtained by replacing all edges of $R$ with copies of the complete bipartite graph $K_{m, m}$, and a sparser graph $G$ is constructed by replacing the edges of $R$ with some $(\varepsilon, \delta)$ super-regular pairs. If a graph $H$ with $\Delta(H) \leq \Delta$ is embeddable into $R(m)$ then it is already embeddable into $G$.

Actually we will need the following consequence of the Blow-up Lemma (where $R$ is a triangle).

Lemma 10. For every $\delta>0$ there exist an $\varepsilon>0$ and $m_{0}$ such that the following holds. Let $G$ be a tripartite graph with tripartition $V(G)=V_{1} \cup V_{2} \cup V_{3}$ such that $\left|V_{1}\right|=\left|V_{2}\right|=\left|V_{3}\right|=m \geq m_{0}$, and let all the 3 pairs $\left(V_{1}, V_{2}\right),\left(V_{1}, V_{3}\right),\left(V_{2}, V_{3}\right)$ be $(\varepsilon, \delta)$-super-regular. Then for every pair of vertices $v_{1} \in V_{1}, v_{2} \in V_{3}$ and for every integer $p, 4 \leq p \leq 3 m, G$ contains an "almost" $P_{p}^{2}$, the square of a path with $p$ vertices connecting $v_{1}$ and $v_{2}$ from which at most two short diagonals are missing.

\footnotetext{
${ }^{1}$ For background, this variant and other variants of the Regularity Lemma see [20].
} 
We emphasize that Lemma 10 is true for any value of $p$ between 4 and $3 m$, not just for the ones that are divisible by 3 . The price we pay is that two short diagonals might be missing which is allowed in our application. Note also that an easier approximate version of this lemma would suffice as well, but for simplicity we use this lemma.

Proof. We think of $G$ as having the orientation $\left(V_{1}, V_{2}, V_{3}\right)$. Because of the Blow-up Lemma it is sufficient to check the statement for the complete tripartite graph (using $r=3$ and $\Delta=4$ in the Blow-up Lemma). We distinguish three cases depending on $p$. If $p=3 k$ for some integer $2 \leq k \leq m$, then we just go around $\left(V_{1}, V_{2}, V_{3}\right) k$ times starting with $v_{1}$ and ending with $v_{2}$, so in this case actually no short diagonal is missing. If $p=3 k+1$ with $k \geq 1$, then we go around $\left(V_{1}, V_{2}, V_{3}\right)(k-1)$ times starting with $v_{1}$, but then in the last round we "turn around", i.e. we finish with vertices $u_{1}, u_{2}, u_{3}, v_{2}$ chosen from $V_{1}, V_{2}, V_{1}, V_{3}$, respectively. Then the only short diagonal missing is between $u_{1}$ and $u_{3}$. Finally, if $p=3 k+2$ with $k \geq 1$, then we go around $\left(V_{1}, V_{2}, V_{3}\right)(k-1)$ times starting with $v_{1}$, but then in the last round we "double up", i.e. we finish with vertices $u_{1}, u_{2}, u_{3}, u_{4}, v_{2}$ chosen from $V_{1}, V_{2}, V_{1}, V_{2}, V_{3}$, respectively. Then the only two short diagonals missing are between $u_{1}$ and $u_{3}$ and between $u_{2}$ and $u_{4}$.

With these preparations now we are ready to prove Theorem 3 from Theorem 7. Let

$$
\varepsilon \ll \eta \ll 1
$$

$m_{0}$ sufficiently large compared to $1 / \varepsilon$ and $M_{0}$ obtained from Lemma 8. Suppose we have a 2-coloring of a complete graph with vertex set $V,|V|=(1+\eta) 7 n / 3$ (for simplicity assume that this is a sufficiently large integer). We apply Lemma 8, We obtain a partition of $V$, that is $V=\cup_{0 \leq i \leq \ell} V_{i}$. We define the following reduced graph $G^{R}$ : The vertices of $G^{R}$ are $p_{1}, \ldots, p_{\ell}$, and there is an edge between vertices $p_{i}$ and $p_{j}$ if the pair $\left(V_{i}, V_{j}\right)$ is $\varepsilon$-regular in both colors. The edge $p_{i} p_{j}$ is colored with the majority color in $K\left(V_{i}, V_{j}\right)$. Thus $G^{R}$ is a $(1-\varepsilon)$-dense 2 -colored graph on $\ell$ vertices. Then we "trim" $G^{R}$ in the standard way: there is a subgraph $H^{R}$ on at least $(1-\sqrt{\varepsilon}) \ell$ vertices where the maximum degree of the complement is less than $\sqrt{\varepsilon} \ell$ (see for example Lemma 9 in [13]). In other words the third color class does not contain a star $K_{1, t}$ with $t=\sqrt{\varepsilon} \ell$, as we need in Theorem 7 .

Applying Theorem 7 to $H^{R}$ with $t=\sqrt{\varepsilon} \ell$, we can get a large monochromatic (say red) connected triangle matching in $H^{R}$ (and thus in $G^{R}$ ). For a triangle $T_{i}, 1 \leq i \leq \ell_{1}$ in this connected triangle matching denote the corresponding clusters by $\left(V_{1}^{i}, V_{2}^{i}, V_{3}^{i}\right)$. Thus (using (11)) we may assume that the number of vertices in the union of these clusters is between $\left(1+\frac{\eta}{2}\right) n$ and $(1+\eta) n$. Next, first using the fact that this is a connected triangle matching we find red connecting paths $P_{i}^{R}$ in $G^{R}$ between $T_{i}$ and $T_{i+1}, 1 \leq i \leq \ell_{1}$ (where $T_{\ell_{1}+1}=T_{1}$ ) and then from these connecting paths $P_{i}^{R}$ we can find vertex disjoint red connecting paths $P_{i}$ in the original graph between a vertex of 
$V_{3}^{i}$ to a vertex of $V_{1}^{i+1}$. This procedure is rather standard by now, see for example [13] for the details.

These connecting paths $P_{i}$ will be part of the final monochromatic $C_{n}^{2, c}$ we are constructing, so on these segments there will not be any short diagonals guaranteed. However, since the paths $P_{i}^{R}$ are in $G^{R}$, their total length is indeed a constant depending on $\varepsilon$ only. We remove the internal vertices of the paths $P_{i}$ from our graph; let us denote their total number by $c_{1}$. Thus on the remaining segments we need exactly $n-c_{1}$ vertices.

Furthermore, we remove some more vertices from each $\left(V_{1}^{i}, V_{2}^{i}, V_{3}^{i}\right), 1 \leq i \leq l_{1}$ to achieve super-regularity in red in all of the three pairs. Finally we remove some more vertices to get a balanced super-regular tripartite graph. The number of remaining vertices in the union of the clusters in the triangles is still between $n$ and $(1+\eta) n$ using (1). For simplicity we still denote the clusters by $V_{j}^{i}$.

Finally we will lift the triangles back to almost square-paths in the original graph using Lemma 10. Let us denote by $\left(1-\eta^{\prime}\right)$ the ratio of $n$ and the number of remaining vertices in the union of the clusters in the triangles, so $0 \leq \eta^{\prime} \ll 1$. Let us use Lemma 10 in each balanced super-regular tripartite graph $\left(V_{1}^{i}, V_{2}^{i}, V_{3}^{i}\right), 2 \leq i \leq l_{1}$ with $p_{i}=\left\lfloor\left(1-\eta^{\prime}\right) 3\left|V_{1}^{i}\right|\right\rfloor$ to connect the two endpoints of the connecting paths $P_{i-1}$ and $P_{i}$ with an almost square-path of length $p_{i}$. Finally we use Lemma 10 one more time in the balanced super-regular tripartite graph $\left(V_{1}^{1}, V_{2}^{1}, V_{3}^{1}\right)$ with a $p_{1}$ value that makes the total length exactly $n$, to connect the two endpoints of the connecting paths $P_{\ell_{1}}$ and $P_{1}$ with an almost square-path of length $p_{1}$. This is possible since this $p$ value is less than $\left\lfloor\left(1-\eta^{\prime}\right) 3\left|V_{1}^{1}\right|\right\rfloor$ only by a constant. Putting together the almost square-paths within the triangles with the connecting paths we get the red almost square-cycle of length $n$ with only a constant number of short diagonals missing.

\section{References}

[1] P. Allen, G. Brightwell, J. Skokan, Ramsey goodness - and otherwise, Combinatorica 33 (2013), pp. 125-160.

[2] J. Balog, J. Barát, D. Gerbner, A. Gyárfás, G. N. Sárközy, Partitioning edge-2colored graphs by monochromatic paths and cycles, to appear in Combinatorica.

[3] S. A. Burr, P. Erdős, J. H. Spencer, Ramsey theorems for multiple copies graphs, Transactions of the American Mathematical Society 209 (1975), pp. 87-99.

[4] P. Chau, L. DeBiasio, H. Kierstead, Pósa's conjecture for graphs of order at least $8 \times 10^{9}$. Random Structures and Algorithms, 39 (4) (2011), pp. 507-525. 
[5] V. Chvatál, V. Rödl, E. Szemerédi, W.T. Trotter Jr., The Ramsey number of a graph with bounded maximum degree, J. Combin. Theory Ser. B 34 (1983), pp. 239-243.

[6] D. Conlon, J. Fox, and B. Sudakov, On two problems in graph Ramsey theory, Combinatorica 32 (2012), pp. 513-535.

[7] K. Corradi, A. Hajnal, On the maximal number of independent circuits in a graph, Acta Math. Acad. Sci. Hungar. 14 (1963), pp. 423-439.

[8] E. J. Cockayne, P. J. Lorimer, The Ramsey number for stripes, J. Austral. Math. Soc. 19 (1975), pp. 252-256.

[9] A. Figaj, T. Luczak, The Ramsey number for a triple of long even cycles, Journal of Combinatorial Theory, Ser. B 97 (2007), pp. 584-596.

[10] L. Gerencsér, A. Gyárfás, On Ramsey type problems, Ann. Univ. Sci. Eötvös, Budapest 10 (1967), pp. 167 - 170.

[11] A. Gyárfás, J. Lehel, G. N. Sárközy, R. H. Schelp, Monochromatic Hamiltonian Berge cycles in colored complete hypergraphs, Journal of Combinatorial Theory, Ser. B 98 (2008), pp. 342-358.

[12] A. Gyárfás, M. Ruszinkó, G. N. Sárközy, E. Szemerédi, An improved bound for the monochromatic cycle partition number, Journal of Combinatorial Theory, Series B 96 (2006), pp. 855-873.

[13] A. Gyárfás, M. Ruszinkó, G. N. Sárközy, E. Szemerédi, Three-color Ramsey numbers for paths, Combinatorica, 27 (2007), pp. 35-69.

[14] A. Gyárfás, M. Ruszinkó, G. N. Sárközy, E. Szemerédi, Partitioning 3-colored complete graphs into three monochromatic cycles, Electronic J. of Combinatorics 18 (2011), N.53.

[15] A. Gyárfás, G. N. Sárközy, Star versus two stripes Ramsey numbers and a conjecture of Schelp, Combinatorics, Probability and Computing 21 (2012), pp. 179186.

[16] A. Gyárfás, G. N. Sárközy, E. Szemerédi, The Ramsey number of diamond matchings and loose cycles in hypergraphs, Electronic Journal of Combinatorics, 15 (2008), R126.

[17] J. Komlós, G. N. Sárközy and E. Szemerédi, On the square of a Hamiltonian cycle in dense graphs, Random Structures and Algorithms 9 (1996), pp. 193-211. 
[18] J. Komlós, G. N. Sárközy, E. Szemerédi, Blow-up Lemma, Combinatorica 17 (1997), pp. 109-123.

[19] J. Komlós, G. N. Sárközy, E. Szemerédi, An algorithmic version of the Blow-up Lemma, Random Structures and Algorithms 12 (1998), pp. 297-312.

[20] J. Komlós and M. Simonovits, Szemerédi's Regularity Lemma and its applications, in: Graph Theory, in Combinatorics, Paul Erdős is Eighty (D. Miklós, V.T. Sós and T. Szőnyi, Eds.), pp. 295-352, Bolyai Society Mathematical Studies, Vol. 2, Budapest, 1996.

[21] I. Levitt, G. N. Sárközy, E. Szemerédi, How to avoid using the Regularity Lemma; Pósa's Conjecture revisited, Discrete Mathematics 310 (2010), pp. 630-641.

[22] T. Łuczak, $R\left(C_{n}, C_{n}, C_{n}\right) \leq(4+o(1)) n$, Journal of Combinatorial Theory, Ser. $B 75$ (1999), pp. 174-187.

[23] E. Szemerédi, Regular partitions of graphs, Colloques Internationaux C.N.R.S. № 260 - Problèmes Combinatoires et Théorie des Graphes, Orsay (1976), pp. 399-401. 